\title{
SIMULAÇÃO DE MONTE CARLO PARA RESOLUÇÃO DE PROBLEMAS DE INTEGRAÇÃO
}

\author{
Vinícius Brum Ferreira \\ Universidade Federal Fluminense/Instituto do Noroeste Fluminense de Educação \\ Superior - (INFES/UFF) \\ Av. João Jasbick, s/nº, Bairro Aeroporto, Santo Antônio de Pádua - RJ \\ brum_vinicius@id.uff.br
}

\section{Thiago Jordem Pereira}

Universidade Federal Fluminense/Instituto do Noroeste Fluminense de Educação Superior - (INFES/UFF)

Av. João Jasbick, s/nº, Bairro Aeroporto, Santo Antônio de Pádua - RJ

tjordem@id.uff.br

\section{RESUMO}

O principal objetivo deste trabalho é estudar a eficiência do método de Monte Carlo na aproximação da solução de alguns problemas complexos de integração unidimensionais e multidimensionais, uma vez que estes tipos de integrais podem ser utilizadas para representar diversos tipos de problemas práticos. Com os resultados numéricos obtidos analisa-se a influência do tamanho da amostra na solução aproximada dos problemas propostos, levando em consideração os erros relativos das aproximações.

Palavra-chave: Método de Monte Carlo; Integrais; Simulação Estocástica.

\begin{abstract}
The main objective of this work is to study the efficiency of Monte Carlo method in approaching the solution of some complex one-dimensional and multidimensional integration problems, since these types of integrals can be used to represent several types of practical problems. With the numerical results obtained, the influence of the sample size on the approximate solution of the proposed problems is analyzed, taking into account the relative errors of the approximations.
\end{abstract}

Keywords: Monte Carlo Method; Integrals; Stochastic Simulation.

\section{Como Citar:}

FERREIRA, Vinícius Brum; PEREIRA, Thiago Jordem. Simulação de Monte Carlo para Resolução de Problemas de Integração. In: SIMPÓSIO DE PESQUISA OPERACIONAL E LOGÍSTICA DA MARINHA, 19., 2019, Rio de Janeiro, RJ. Anais [.. . ]. Rio de Janeiro: Centro de Análises de Sistemas Navais, 2019. 


\section{INTRODUÇÃO}

Sabe-se que o conceito de integração pode ser utilizado para resolver problemas que envolvem cálculos de áreas, volumes, comprimentos de curvas, centro de massa, momento de inércia, esperança estatística, predições populacionais, força sobre um dique e muitos outros [1, 2, 4, 5, 6, 8, 10]. As soluções destes problemas podem ser obtidas por diversas técnicas de integração, que por sua vez utilizam procedimentos analíticos para encontrar as antiderivadas das funções. No entanto, em alguns problemas de integração, as antiderivadas das funções podem ser difíceis ou impossíveis para se obterem, pois as técnicas analíticas podem não fornecer suporte adequado para os tratamentos das funções dos problemas de integração [10]. Neste caso, os método numéricos de integração tornam-se alternativas atrativas e eficientes [8, 9].

Dentre os diversos métodos de integração [1, 9] existentes pode-se destacar o método de Monte Carlo que teve sua primeira publicação feita por Metropolis e Ulam em 1949 [7]. O método de Monte Carlo baseia-se em técnicas de amostragens aleatórias, nas quais geram-se amostras a partir de uma distribuição de probabilidades para aproximar problemas de natureza determinística ou estocástica [7, 8]. Este método numérico tem sido bastante utilizado para resolver problemas complexos de integração e calcular áreas de regiões formadas por figuras cujas funções matemáticas são desconhecidas.

O principal objetivo deste trabalho de pesquisa é estudar a eficiência do método de Monte Carlo na aproximação de algumas integrais unidimensionais e multidimensionais, uma vez que estes tipos de integrais podem ser utilizadas para representar diversos tipos de problemas práticos. Resultados numéricos são obtidos e apresentados para diversos conjuntos de amostras, onde se analisa as quantidades de amostras geradas e os erros relativos das aproximações.

\section{O MÉTODO DE MONTE CARLO}

\subsection{INTEGRAÇÃO UNIDIMENSIONAL}

Para uma introdução ao método de Monte Carlo (MC) na resolução de problemas de integração, considera-se que o problema de interesse é aproximar a integral unidimensional do tipo [1, 8]

$$
I=\int_{0}^{1} g(x) d x
$$

onde $g(x): \mathbb{R} \rightarrow \mathbb{R}$ é a função de interesse e $x \in[0,1]$. Suponha que $U$ é uma variável aleatória uniformemente distribuída no intervalo [0,1], então o valor esperado da variável aleatória $g(U)$ é dado por [1, 8] 


$$
E(g(U))=\int_{0}^{1} g(x) f_{U}(x) d x=\int_{0}^{1} g(x) d x=I,
$$

onde $f_{U}(x)$ é uma função de densidade de probabilidades definida por

$$
f_{U}(x)=\left\{\begin{array}{lll}
1, & \text { se } x \in[0,1] \\
0, & \text { se } \text { caso contrário }
\end{array}\right.
$$

Suponha que $U_{1}, U_{2}, \cdots, U_{n}$ são variáveis aleatórias independentes e uniformementes distribuídas no intervalo [0,1]. Portanto, o método MC aproxima a integral (1) pela média amostral [1, 8 ]

$$
\hat{I}=\frac{1}{n} \sum_{i=1}^{n} g\left(U_{i}\right)
$$

ou seja,

$$
\int_{0}^{1} g(x) d x=E(g(U)) \simeq \frac{1}{n} \sum_{i=1}^{n} g\left(U_{i}\right) .
$$

Tem-se que a geração da amostra $\left\{U_{i}\right\}_{i=1}^{n}$ é independente, seguindo pela Lei Forte dos Grande Números $\hat{I}$ vai convergir para $E(g(U))$, ou seja,

$$
\hat{I}=\frac{1}{n} \sum_{i=1}^{n} g\left(U_{i}\right) \underset{n \rightarrow \infty}{\stackrel{q . c .}{\rightarrow}} I=E(g(U)) .
$$

Geralmente os limites de integração não são iguais a 0 e 1, e por esta razão deve-se realizar substituições de variáveis. A seguir, mostra-se os processos de substituições de variáveis para os problemas de integração definida e imprópria.

\subsubsection{INTEGRAÇÃO DEFINIDA UNIDIMENSIONAL}

Para um problema de integração definida $\int_{a}^{b} f(x) d x$, faz-se

$$
y=\frac{(x-a)}{(b-a)} \quad \text { e } \quad d y=\frac{d x}{(b-a)}
$$

Assim, 


$$
I=\int_{0}^{1} f(a+[b-a] y)(b-a) d y=\int_{0}^{1} h(y) d y
$$

onde $h(y)=(b-a) f(a+[b-a] y)$.

\subsubsection{INTEGRAÇÃO IMPRÓPRIA UNIDIMENSIONAL}

Para a integral imprópria do tipo $\int_{0}^{\infty} f(x) d x$, aplica-se a seguinte substituição de variáveis

$$
y=\frac{1}{(x+1)} \quad e \quad d y=\frac{-d x}{(x+1)^{2}}=-y^{2} d x
$$

Assim,

$$
I=\int_{0}^{1} h(y) d y
$$

onde $h(y)=\frac{f\left(\frac{1}{y}-1\right)}{y^{2}}$

Em ambas substituições de variáveis, aproximar-se $I$ gerando continuamente números aleatórios $y_{i} \sim \mathbb{U}[0,1]$ e tomando o valor médio de $h\left(y_{i}\right)$, com $i=0,1,2, \cdots, n$, onde $n$ é o tamanho da amostra. Assim,

$$
\hat{I}=\frac{1}{n} \sum_{i=1}^{n} h\left(y_{i}\right)
$$

\subsection{INTEGRAÇÃO MULTIDIMENSIONAL}

Pode-se utilizar o método de Monte Carlo na aproximação de problemas de integração multidimensionais do tipo

$$
J=\int_{0}^{1} \cdots \int_{0}^{1} f\left(x_{1}, \cdots, x_{m}\right) d x_{1} \cdots d x_{m}
$$

onde $f(x): \mathbb{R}^{m} \rightarrow \mathbb{R}$ é a função $m$-dimensional de interesse e $x_{1}, \cdots, x_{m} \in$ $[0,1]$. Suponha que $U_{1}, \cdots, U_{m}$ são variáveis aleatórias independentes e uniforme- 
mentes distribuídas no intervalo [0,1], então o valor esperado das variáveis aleatórias $f\left(U_{1}, \cdots, U_{m}\right)$ é dado por [8]

$$
E\left(f\left(U_{1}, \cdots, U_{m}\right)\right)=J
$$

Neste caso, precisa-se gerar $k$ conjuntos independentes de $U_{1}, \cdots, U_{m}$, isto é,

$$
\left\{U_{1}^{1}, \cdots, U_{m}^{1}\right\} ;\left\{U_{1}^{2}, \cdots, U_{m}^{2}\right\} ;\left\{U_{1}^{3}, \cdots, U_{m}^{3}\right\} ; \cdots ;\left\{U_{1}^{k}, \cdots, U_{m}^{k}\right\}
$$

onde $i=1, \cdots, k$. Portanto, o método MC aproxima a integral (12) pela média amostral [8]

$$
\hat{J}=\frac{1}{k} \sum_{i=1}^{k} f\left(U_{1}^{1}, \cdots, U_{m}^{1}\right),
$$

onde as variáveis aleatórias $f\left(U_{1}^{i}, \cdots, U_{m}^{i}\right)$ são independentes e identicamentes distribuídas (i.i.d.).

\section{RESULTADOS NUMÉRICOS}

Nesta seção, apresenta-se os resultados numéricos obtidos pelo método de Monte Carlo para resolução de seis problemas de integração, com diferentes limites de integração, sendo dois problemas de integração definida (Subseção 3.1), dois problemas de integração imprópria (Subseção 3.1) e dois problemas de integração multidimensional (Subseção 3.1). Em todos os estudos numéricos analisa-se as quantidades de amostras geradas e os erros relativos das aproximações.

\subsection{RESULTADOS NUMÉRICOS 1}

Como motivação inicial considera-se as integrais unidimensionais definidas $I_{1}$ e $I_{2}$, fazendo o uso das transformações apresentadas em 2.1.1. Sabe-se que as soluções analíticas dos problemas $I_{1}$ e $I_{2}$ são 0,8104970 [4] e 3,6275987 [4], respectivamente. Desta forma, para se analisar a eficiência do método de Monte Carlo para a aproximação dos referidos problemas, foram geradas amostras com 10, 100, 250, 500, 1.000, 5.000, 10.000 e 100.000 elementos, onde foram calculados os erros relativos para cada amostra gerada (veja a Tabela 1).

Pode-se observar nos dados apresentados na Tabela 1 que o método MC conseguiu obter as soluções aproximadas dos problemas $I_{1}$ e $I_{2}$. Nota-se que as quantidades de elementos das amostras influenciam nos resultados obtidos, onde há uma variação significativa dos erros relativos. Para o conjunto de amostras com 10 elementos, o método de Monte Carlo obteve um erro relativo de 2,584\% 
e 10,979\% na aproximação dos resultados dos problemas $I_{1}$ e $I_{2}$, respectivamente. Nota-se também que, ao aumentar o tamanho da amostra para 100.000 elementos, o erro relativo reduz para $0,001 \%$ e $0,023 \%$ na aproximação dos resultados dos problemas $I_{1}$ e $I_{2}$, respectivamente. De fato, quanto maior é o numéro de elementos de uma amostra as estimativas de $\hat{I}_{1}$ e $\hat{I}_{2}$ convergem quase certamente para as seus respectivos valores esperados, em conformidade com o que foi apresentado na igualdade (6). Nas Figuras 1 e 2 apresenta-se, respectivamente, os gráficos dos problemas $I_{1}$ e $I_{2}$, gerados com 100 e 100.000 elementos.

Vale a pena ressaltar que para o problema $I_{1}$, onde se utiliza uma amostra com tamanho de 5.000 elementos, o método de Monte Carlo obteve um erro relativo de $0,009 \%$, que por sua vez possui a mesma ordem de grandeza do erro relativo obtido para um conjunto de 100.000 elementos. Desta forma, pode-se dizer que uma amostra com tamanho de 5.000 elementos foi suficiente para o método de Monte Carlo obter excelente aproximação do problema de integração $I_{1}$.

Table 1: Resultados dos problemas de integração definida $I_{1}$ e $I_{2}$.

\begin{tabular}{c|c|c|c|c}
\hline & \multicolumn{2}{|c|}{$I_{1}=\int_{0}^{\frac{2 \pi}{6}} \frac{1}{\sin (x)+\cos (x)} d x$} & \multicolumn{2}{c}{$I_{2}=\int_{-\pi}^{\pi} \frac{1}{2+\sin (x)} d x$} \\
\hline $\begin{array}{c}\text { Tamanho da } \\
\text { Amostra }\end{array}$ & $\hat{I}_{1}-\mathrm{MC}$ & Erro Relativo & $\hat{I}_{2}-\mathrm{MC}$ & Erro Relativo \\
\hline 10 & 0,7895543 & $2,584 \%$ & 4,0258580 & $10,979 \%$ \\
\hline 100 & 0,8142427 & $0,462 \%$ & 3,6838240 & $1,550 \%$ \\
\hline 250 & 0,8003836 & $1,248 \%$ & 3,8377220 & $5,792 \%$ \\
\hline 500 & 0,8127465 & $0,278 \%$ & 3,5671770 & $1,666 \%$ \\
\hline 1.000 & 0,8109047 & $0,050 \%$ & 3,6125540 & $0,415 \%$ \\
\hline 5.000 & 0,8105686 & $0,009 \%$ & 3,6359110 & $0,229 \%$ \\
\hline 10.000 & 0,8105474 & $0,006 \%$ & 3,5965580 & $0,856 \%$ \\
\hline 100.000 & 0,8105044 & $0,001 \%$ & 3,6267670 & $0,023 \%$ \\
\hline
\end{tabular}

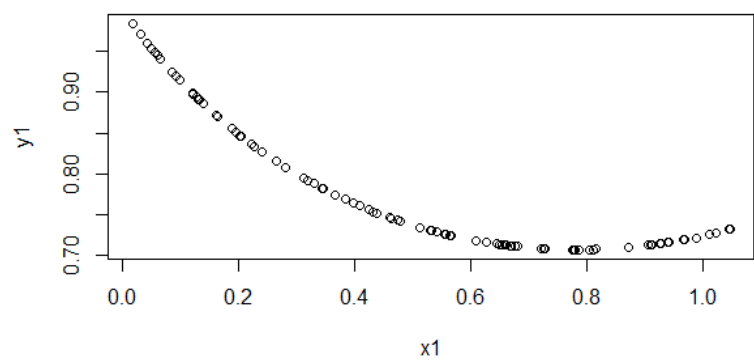

(a) 100 amostras.

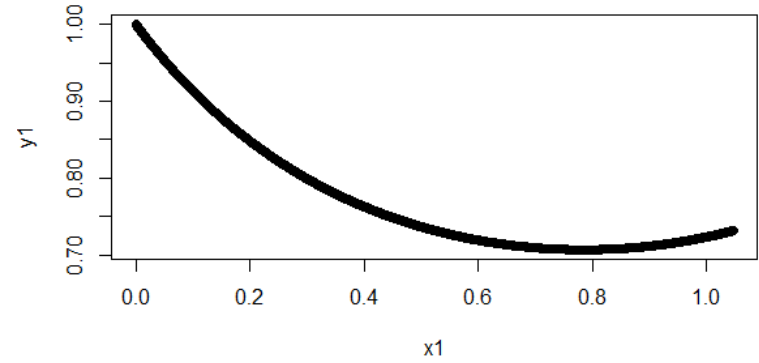

(b) 100.000 amostras.

Figure 1: Gráficos da integral definida $I_{1}$. 


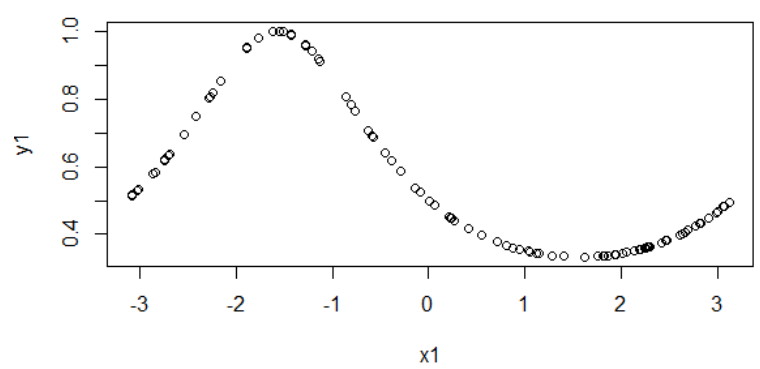

(a) 100 amostras.

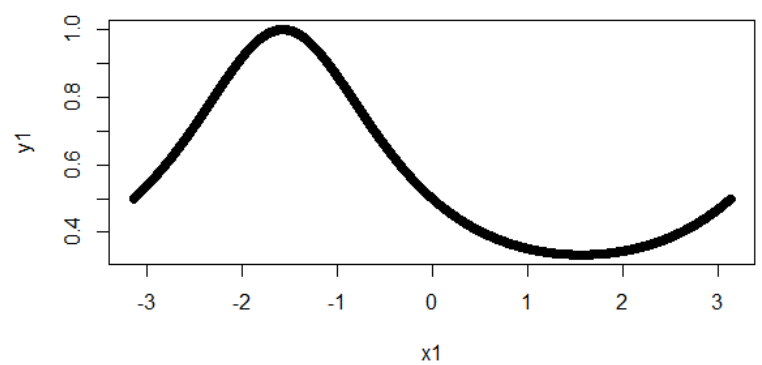

(b) 100.000 amostras.

Figure 2: Gráficos da integral definida $I_{2}$.

\subsection{RESULTADOS NUMÉRICOS 2}

Os resultados apresentados na Tabela 2 são semelhantes aos apresentados na Tabela 1 da Subseção 3.1. As diferenças estão nos problemas de integração, onde se utiliza as integrais impróprias $I_{3}$ e $I_{4}$ com soluções analíticas $I_{3}=0,3490659$ [10] e $I_{4}=0,3926991$ [10], respectivamente. Desta forma, nota-se que para os problemas propostos o método de Monte Carlo também obteve resultados numéricos próximos dos resultado analíticos. Para o problema $I_{3}$, onde se utiliza uma amostra com 5.000 elementos, o método obteve um erro relativo de $0,571 \%$. Já para o problema $I_{4}$, o erro relativo foi de $0,821 \%$. Observa-se também que para 100 amostras o problema $I_{4}$ chegou em uma boa aproximação do problema, obtendo um erro relativo de 0,007\%. Porém, como o tamanho da amostra foi de apenas 100 elementos, sugere-se que outros estudos também sejam feitos, com o objetivo investigar o comportamento do referido resultado. Nas Figuras 3 e 4 apresenta-se, respectivamente, os gráficos dos problemas $I_{3}$ e $I_{4}$, gerados com 100 e 100.000 amostras.

Table 2: Resultados dos problemas de integração $I_{3}$ e $I_{4}$.

\begin{tabular}{c|c|c|c|c}
\hline & \multicolumn{2}{|c|}{$I_{3}=\int_{-\infty}^{\infty} \frac{x^{2}}{9+x^{6}} d x$} & \multicolumn{2}{c}{$I_{4}=\int_{0}^{\infty} \frac{x \arctan (x)}{1+x^{2}} d x$} \\
\hline $\begin{array}{c}\text { Tamanho da } \\
\text { Amostras }\end{array}$ & $\hat{I}_{3}-\mathrm{MC}$ & Erro Relativo & $\hat{I}_{4}-\mathrm{MC}$ & Erro Relativo \\
\hline 10 & 0,5284177 & $51,381 \%$ & 0,4062322 & $3,446 \%$ \\
\hline 100 & 0,3362064 & $3,684 \%$ & 0,3926712 & $0,007 \%$ \\
\hline 250 & 0,3809246 & $9,127 \%$ & 0,3765451 & $4,114 \%$ \\
\hline 500 & 0,3859897 & $10,578 \%$ & 0,3995221 & $1,737 \%$ \\
\hline 1.000 & 0,3362064 & $3,684 \%$ & 0,4026397 & $2,531 \%$ \\
\hline 5.000 & 0,3510603 & $0,571 \%$ & 0,3894745 & $0,821 \%$ \\
\hline 10.000 & 0,3484699 & $0,171 \%$ & 0,3950380 & $0,596 \%$ \\
\hline 100.000 & 0,3489704 & $0,027 \%$ & 0,3923575 & $0,087 \%$ \\
\hline
\end{tabular}




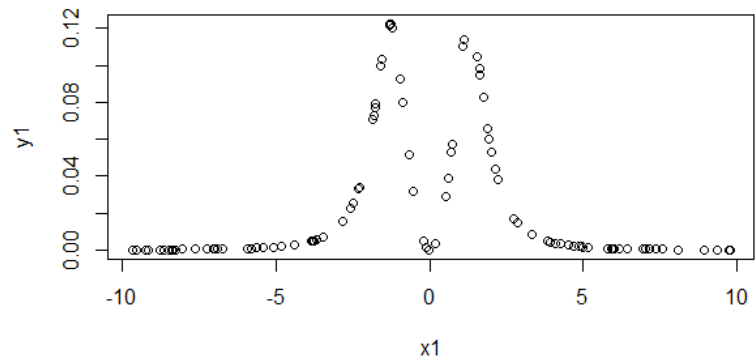

(a) 100 amostras.

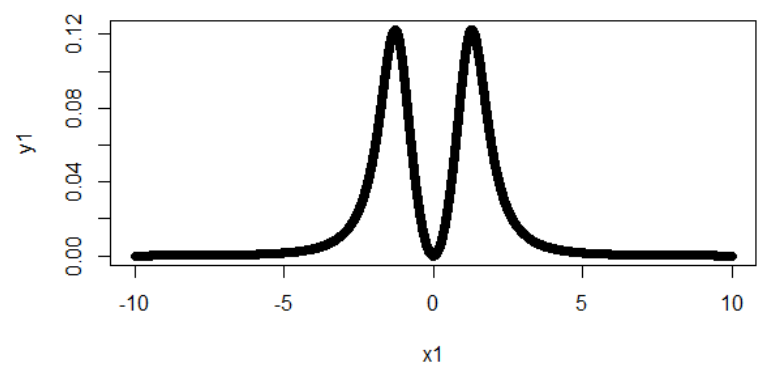

(b) 100.000 amostras.

Figure 3: Gráficos da integral $I_{3}$.

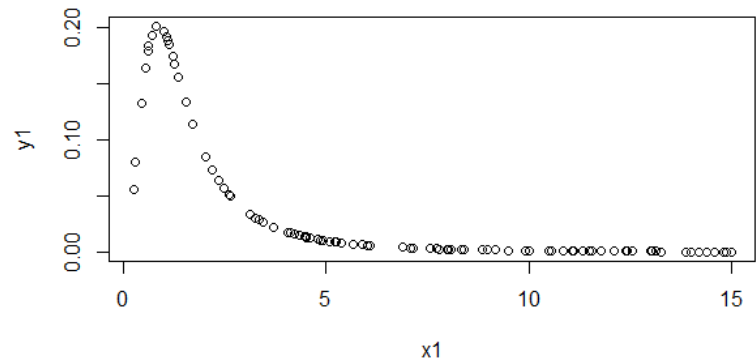

(a) 100 amostras.

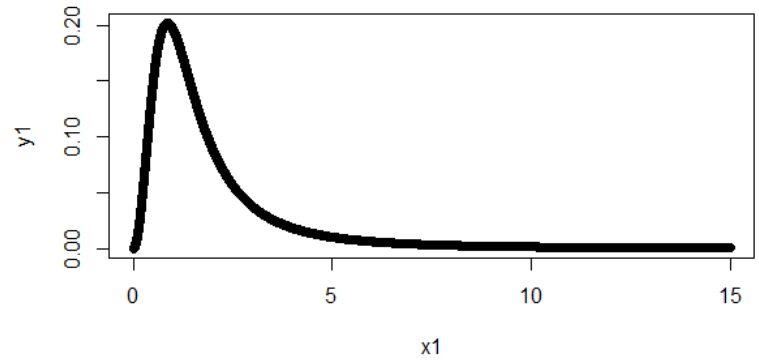

(b) 100.000 amostras.

Figure 4: Gráficos da integral $I_{4}$.

\subsection{RESULTADOS NUMÉRICOS 3}

Agora, apresenta-se os resultados numéricos obtidos pelo método de Monte Carlo para a resolução das integrais multidimensionais $I_{5}$ e $I_{6}$, com soluções analítica $I_{5}=21,5[\underline{6}]$ e $I_{6}=\frac{3 \pi}{4}$ [6], respectivamente. Mais uma vez, percebe-se que método de Monte Carlo obteve resultados satisfatórios (veja a Tabela 3). Observa-se que para uma amostra de 5.000 elementos, os erros relativo dos problemas $I_{5}$ e $I_{6}$ foram de $0,048 \%$ e $3,459 \%$, respectivamente. Pode-se observar na Figura 5 as diferenças entre os gráficos do problema $I_{5}$, gerado com amostras de 100 e 100.000 elementos. 
Table 3: Resultados dos problemas de integração multidimensional $I_{5}$ e $I_{6}$.

\begin{tabular}{c|c|c|c|c}
\hline & \multicolumn{2}{|c|}{$I_{5}=\int_{0}^{3} \int_{0}^{2}\left(4-\frac{1}{9} x^{2}-\frac{1}{16} y^{2}\right) d y d x$} & \multicolumn{2}{|c}{$I_{6}=\int_{0}^{\frac{\pi}{2}} \int_{\frac{\pi}{6}}^{\frac{\pi}{2}} \int_{0}^{2} \rho^{3} \sin (\phi) \cos (\phi) d \rho d \phi d \Theta$} \\
\hline $\begin{array}{c}\text { Tamanho da } \\
\text { Amostras }\end{array}$ & $\hat{I}_{5}-\mathrm{MC}$ & Erro Relativo & $\hat{I}_{6}-\mathrm{MC}$ & Erro Relativo \\
\hline 10 & 21,1273000 & $1,733 \%$ & 2,8587130 & $21,328 \%$ \\
\hline 100 & 21,3955900 & $0,486 \%$ & 2,1798560 & $7,484 \%$ \\
\hline 250 & 21,5995200 & $0,463 \%$ & 2,5205810 & $6,977 \%$ \\
\hline 500 & 21,6059500 & $0,493 \%$ & 2,0995640 & $10,892 \%$ \\
\hline 1.000 & 21,5233300 & $0,109 \%$ & 2,5869500 & $9,794 \%$ \\
\hline 5.000 & 21,4896200 & $0,048 \%$ & 2,2747020 & $3,459 \%$ \\
\hline 10.000 & 21,4926300 & $0,034 \%$ & 2,3505070 & $0,241 \%$ \\
\hline 100.000 & 21,4999200 & $0,000 \%$ & 2,3581080 & $0,081 \%$ \\
\hline
\end{tabular}

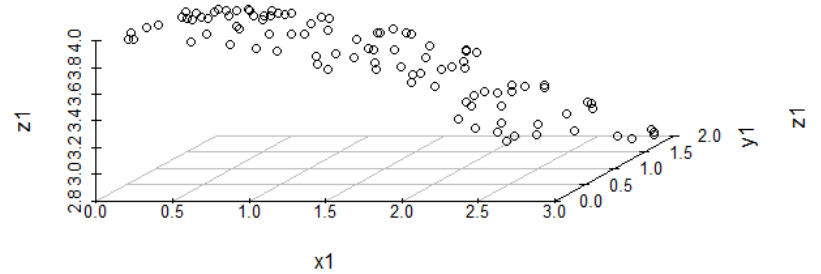

(a) 100 amostras.

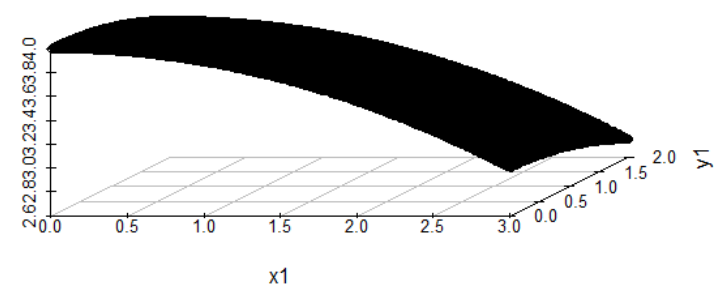

(b) 100.000 amostras.

Figure 5: Gráficos da integral $I_{5}$.

\section{CONCLUSÕES}

Com os resultados numéricos obtidos neste trabalho pode-se dizer que o método de Monte Carlo se mostrou eficiente para resolução de todos os problemas de integração, onde forneceu valores numéricos bem próximos dos valores analíticos. Os resultados obtidos mostraram que o número de amostras geradas influenciam diretamente nas soluções dos problemas propostos. Portanto, pode-se observar que o método de Monte Carlo pode ser uma ferramenta bastante eficiente para a resolução de diversos problemas práticos. 


\section{REFERÊNCIAS BIBLIOGRÁFICAS}

[1] Andrieu, C. and Freitas, N. D. and Doucet, A. and Jordan, M. I. 2003. An Introduction to MCMC for Machine Learning, Machine Learning, $\mathrm{n}^{\circ}$ 50, pp. 5-43. 2, 3

[2] Bussab, Wilton de O. and Merettin, Pedro A. Estatística Básica. 5a . ed. São Paulo: Saraiva, 2004. 2

[3] Gilat, AmosPozo. Métodos numéricos para engenheiros e cientistas. Porto Alegre: Bookman, 2008.

[4] Guidorizzi, Hamilton Luiz. Um curso de cálculo. LTC, 2005. v. 1. 2, 5

[5] Leithold, Louis. O cálculo com Geometria Analítica. $3^{\mathrm{a}}$ ed. Harbra, 1994. v. 1. 2

[6] Leithold, Louis. O cálculo com Geometria Analítica. $3^{\text {a }}$ ed. Harbra, 1994. v. 2. 2., 8

[7] Metropolis, N. and Ulam, S. 1949. The Monte Carlo Method, Journal of the American Statistical Association, v. 44, n 247, pp. 335-341. 2

[8] Robert, C. P. and Casella, G. 2002.Monte Carlo Statistical Methods. 2a ed., v.1, USA: Springer Texts in Statistics. 2, 3, 5

[9] Ruggiero, Márcia A.Gomes e Lopes, Vera Lúcia R. Cálculo Numérico: Aspectos Teóricos e Computacionais. Editora: Pearson Universidades; $2^{\mathrm{a}}$ ed. Rio de Janeiro, 2000. 2

[10] Stewart, James. Cálculo. $7^{\mathrm{a}}$ ed. São Paulo: Cengage Learning, 2013. v. 1. 2 , 7 